\title{
Article \\ Digital Twin-Driven Mating Performance Analysis for Precision Spool Valve
}

\author{
Wenbin Tang ${ }^{1,2,3, * \mathbb{C}}$, Guangshen $\mathrm{Xu}{ }^{1}$, Shoujing Zhang ${ }^{1}$, Shoufeng Jin ${ }^{1}$ and Runxiao Wang ${ }^{3}$ \\ 1 School of Mechanical and Electrical Engineering, Xi'an Polytechnic University, Xi'an 710048, China; \\ xugs988@126.com (G.X.); zhangshoujing@xpu.edu.cn (S.Z.); jdxyjsf@126.com (S.J.) \\ 2 State Key Laboratory for Manufacturing Systems Engineering, Xi'an Jiaotong University, Xi'an 710054, China \\ 3 School of Mechanical and Electrical Engineering, Northwestern Polytechnical University, Xi'an 710072, China; \\ wangrx_nwpu@126.com \\ * Correspondence: tangwb@xpu.edu.cn
}

check for updates

Citation: Tang, W.; Xu, G.; Zhang, S.; Jin, S.; Wang, R. Digital Twin-Driven Mating Performance Analysis for Precision Spool Valve. Machines 2021, 9, 157. https://doi.org/10.3390/ machines 9080157

Academic Editor: Angelos P.

Markopoulos

Received: 7 July 2021

Accepted: 5 August 2021

Published: 7 August 2021

Publisher's Note: MDPI stays neutral with regard to jurisdictional claims in published maps and institutional affiliations.

Copyright: (c) 2021 by the authors. Licensee MDPI, Basel, Switzerland. This article is an open access article distributed under the terms and conditions of the Creative Commons Attribution (CC BY) license (https:// creativecommons.org/licenses/by/ $4.0 /)$.

\begin{abstract}
The precision spool valve is the core component of the electro-hydraulic servo control system, and its performance has an important influence on the flight control of aviation and aerospace products. The non-uniform surface topography error causes a non-uniform mating gap field inside the spool valve, which causes oil leakage and leads to deterioration of the spool valve performance. However, the current oil leakage calculation method only considers the influence of size errors, which is not comprehensive. Thus, how to characterize the mating behavior of the spool valve and its effect on oil leakage with consideration of surface topography errors is the key to evaluating the performance of the spool valve. This paper proposes a new way of analyzing the mating performance of precision spool valves, which considers the surface topography errors based on digital twin technology. Firstly, a general framework for the analysis of mating performance of precision spool valve based on a digital twin is proposed. Then, key technologies of assembly interface geometry modeling, matching behavior modeling and performance analysis are studied. Finally, a quantitative correlation between the mating parameters and the oil leakage of the precision spool valve is revealed. The method is tested on a practical case. This proposed method can provide theoretical support for the accurate prediction and evaluation of the mating performance of the precision spool valve.
\end{abstract}

Keywords: digital twin; precision spool valve; mating performance; surface topography

\section{Introduction}

As the core component of the electro-hydraulic servo control system, precision spool valves are extensively applied to the flight control systems of military products such as aircrafts, rockets and missiles. The performance and reliability of precision spool valves determine the performance and safety of the flight control system and even the entire aircraft. From the perspective of structure and function, a precision spool valve is a hole formed by the valve spool and valve sleeve through precision assembly, with the general assembly accuracy at the magnitude. The precision spool valve regulates the flow and pressure of the hydraulic oil and forms a pressure difference at both ends of the valve spool, which drives linear reciprocating motion of the valve spool inside the valve sleeve, thereby achieving control functions such as electro-hydraulic conversion, power amplification and speed adjustment [1]. Because of processing errors, the assembly interface between the valve spool and the valve sleeve is a random and irregular geometrical surface with various error components such as roughness, waviness and form error (collectively referred to as surface topography errors) rather than an ideal geometrical surface [2]. These uneven surface topography errors affect the assembly performance of the valve spool and valve sleeve, which leads to a non-uniform gap field inside the assembly surface couple, thereby forming a geometrical path for oil leakage. Once there is a pressure difference between the two ends of the gap, the oil in the gap will flow and leak, which will directly affect the 
performance parameters of the spool valve, e.g., control accuracy and frequency response characteristics [3]. Moreover, due to the non-uniform gap error in the assembly surface couple, there will also be a coaxial error between the valve spool and the valve sleeve, which may jam and abnormally wear the spool valve. Hence, analyzing the assembly performance of the precision spool valve and obtaining the characteristics of oil leakage caused by the assembly clearance is of great theoretical and application value to the design and manufacturing of the precision spool valve.

By analyzing the incentives, it is found that the non-uniform geometrical error transforms the macroscopic and idealized assembly constraints in the spool valve into a more complicated microscopic form with errors, which deteriorates the assembly performance of the spool valve and thereby leads to oil leakage. Therefore, the key to studying the assembly performance of spool valve is to accurately characterize errors on the geometrical surface and microscopic geometrical mating. In recent years, there has been a research upsurge of surface geometrical error modeling in the field of 3D tolerance analysis. Modeling methods such as the parametric surface deformation method [4,5], skin model shapes [6-8] and fractal geometrical method [2,3] have emerged one after another, contributing to the rapid development of the integrated expression of various geometrical errors. In particular, the development of precision measurement technology makes it possible to achieve reverse modeling of the geometrical structure of real surfaces based on high-precision measurement data $[9,10]$. On this basis, researchers began to pay attention to the influence of uneven surface topography errors on assembly constraints. This research established the relationship between geometrical contact and assembly positioning of non-ideal surfaces, and analyzed the assembly accuracy using the constrained complex spatial surface registration algorithm $[11,12]$. This research plays an important role in supporting and promoting the study on the mating state inside the spool valve. Mondal et al. [13] analyzed the influence of uncertain dimensions, radial clearance and overlap on the leakage flow of spool valve. In the proposed method, CFD is used to explore uncertain dimensions of the valve. Afatsun et al. [14] proposed a mathematical model for the simulation of flow rate and chamber pressures in spool valves. The ultimate aim of this study is to fulfill the need for a model to precisely determine the geometrical tolerances of spool valve components for optimum performance. The above two representative studies both assume the mating surface of spool valve is an ideal cylindrical surface, which does not consider the impact of shape error. However, the precision spool valve is small-sized with high assembly accuracy, so the surface shape error is an influencing factor that cannot be ignored. Qiu et al. [2] proposed a theory and method of hybrid dimensional part surface to characterize surface topography on multiple scales, and studied the method to predict the amount of oil leaking through precision assembly parts, considering the microscopic topography. However, the parameters with a hybrid dimensional part surface in this paper are random, which means that tiny differences may greatly change the surface topography, thereby affecting the accurate prediction of the actual situation. Wang et al. [15] constructed the real shape of the sealing plane using high-precision point clouds, applied the registration algorithm to analyze the assembly and positioning of the sealing plane with errors and simulated the leakage channel on the sealing plane, so as to evaluate the assembly quality of the sealing plane. This paper took high-precision measured data into consideration, but only for planar features. In summary, researchers have studied the modeling of surface topography and analyzed oil leakage in the spool valve, which provides a theoretical method with reference value for analyzing the assembly performance of spool valves. Nevertheless, the following questions remain to be further answered: (1) How to construct a geometrical model of the real assembly interface of the spool valve to meet the requirements of analyzing its assembly performance? (2) How to characterize the true mating behavior of the spool valve and obtain the non-uniform assembly clearance field and parameters? (3) How to quantify the influence of mating behavior (or mating parameters) on oil leakage?

The digital twin technology has provided new ideas of solving the above-mentioned problems. The core concept of the digital twin technology is to create virtual models of phys- 
ical entities through digitalization, and simulate the behaviors of physical entities based on real-time and historical data $[16,17]$. The multi-disciplinary, multi-scale, multi-probability and high-fidelity digital twin technology facilitates the interaction and integration of physical space and virtual space, which avoids inaccurate analysis and prediction caused by the inconsistency between virtual and real states [18-20]. Currently, studies have been carried out on the application of the digital twin technology in product design [21], process planning [22,23], dynamic scheduling [24,25] and quality monitoring [26,27]. Instructive research results have been obtained, but the digital twin technology is still rapidly developing. The research findings closely correlated with this paper mainly focus on surface topography modeling, assembly error analysis and assembly performance analysis based on a digital twin. There is the following representative research. Söderberg et al. [27] proposed to establish the part error model based on digital twin data to guarantee the assembly accuracy of individualized products online and in real time. Schleich et al. [28] suggested building a digital twin model of the parts based on the skin model, so as to connect design and manufacturing and achieve the interaction between the virtual world and the physical world. Bao et al. [10] proposed a digital twin-driven high-precision method of assembling and adjusting products, constructed a digital model of physical entities based on digital twin data and considered the influences of multi-disciplinary factors in the high-precision product assembly and performance analysis. To sum up, the digital twin technology, with its features such as virtual-real fusion and virtual-real homomorphic mapping, offers a new framework and ideas for characterizing the behaviors of physical products and improving the accuracy of product performance analysis. Nonetheless, targeted research remains to be carried out to solve specific problems.

By summarizing the literature, it can be found that the current research mainly focuses on the influence of size or dimensional errors on the mating performance of precision spool valves, while the research on the influence of surface topography errors on the mating performance of precision spool valves is not thorough enough. One of the difficulties is to accurately construct the real surface topography and predict oil leakage based on the real surface topography. Digital twin technology provides a framework that integrates the real world and the virtual world, and can truly reflect the characteristics and behavior of physical objects in the virtual space. It is a new attempt to introduce digital twin technology into the analysis of the mating performance of the precision spool valve. With the precision spool valve as the research object, this paper proposed an overall framework of analyzing assembly performance of precision spool valves based on a digital twin, modeled geometrical topography of the assembly interface, modeled the assembly behavior of precision spool valve, analyzed the mating performance of precision spool valve and established a quantitative correlation between the assembly parameters and oil leakage of the precision spool valve. The proposed method may provide a theoretical basis for accurately predicting and evaluating the assembly performance of the precision spool valve.

\section{Overall Framework of Analyzing Mating Performance of Precision Spool Valves Based on Digital Twin}

According to the five-dimensional structure framework of the digital twin proposed by Tao et al. [18], the digital twin system consists of physical space entities, the digital twin model, service system, digital twin data and link. Guided by this framework, this paper put forward an overall framework of analyzing mating performance of precision spool valves based on the digital twin, as shown in Figure 1. This framework is composed of five parts: physical spool valve, digital twin spool valve, system service, digital twin data and link. The physical spool valve, as a physical object in the physical space, possesses a real surface reflecting the machining error, real geometrical movements and sealing characteristics. The actual surface error and oil leakage value can be obtained by means of precision measurement and flow leakage detection. The digital twin spool valve, as a mapping of the physical spool valve in the virtual space, truthfully reveals the geometrical and physical behaviors of the physical spool valve. In this paper, the system service is concretized as predicting the leakage characteristics of spool valve, that is, analyzing 
the mating performance of the spool valve using the physical spool valve, digital twin spool valve and relevant data. The digital twin data refers to all the data associated with the digital twin, which are used to realize the fusion of virtual space and real space, the interaction of virtual space and real space and the prediction and analysis of real behavior of real objects in the real word $[18,22]$. In this paper, it refers to the actually measured data and calculation data gained from the physical spool valve and the digital twin spool valve to support the virtual-real interaction process. The link (the broken lines with arrows in the figure) connects the above four parts into an organic whole, so that different parts can interact with each other. To support virtual-real fusion and homomorphic mapping, this paper focused on the following aspects:

Physical Spool Valve

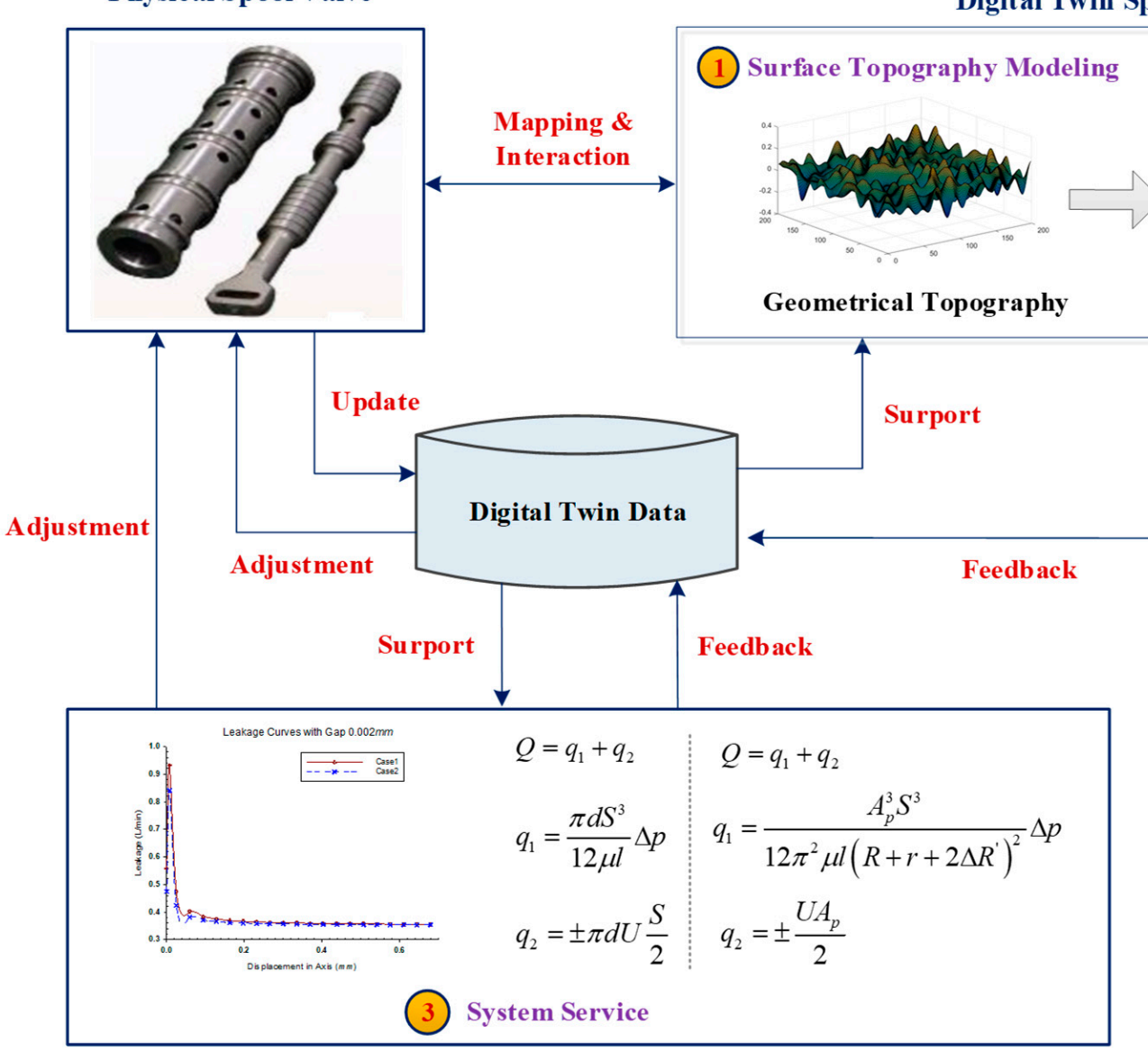

Digital Twin Spool Valve

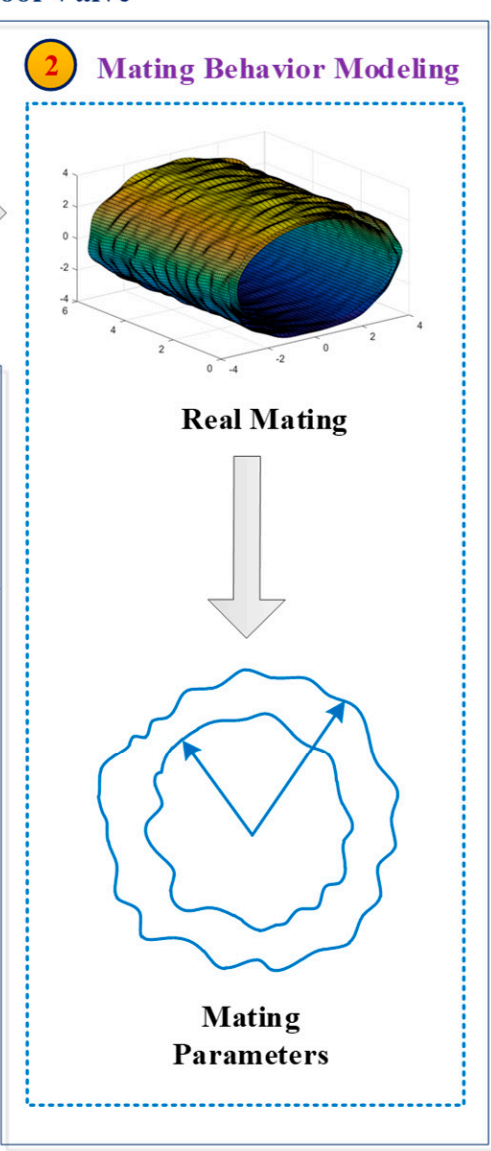

Figure 1. Overall framework of this paper.

(1) With design tolerance as the basis, this paper constructed an assembly interface of precision spool valves that could characterize actual errors. The microscopic topography of the cylindrical assembly interface between the valve spool and the valve sleeve is the key factor affecting the assembly clearance and oil leakage of the precision spool valve. However, the theoretical CAD model, the only model available in the design stage, is unable to reflect the complex geometrical topography of the interface. Although size and form tolerances will be assigned to the assembly interface and related geometrical features in the design stage, these tolerances only reflect the allowable error range, namely the tolerance zone, of the assembly interface after machining rather than manifest the specific topography features. Therefore, it is of necessity to combine the CAD model and design tolerance information to establish a geometrical model of the assembly interface topography that can reveal the actual error, so as to lay the foundation for establishing the digital twin spool valve. 
(2) Based on the geometrical model of the assembly interface topography, this paper analyzed the mating behavior of the precision spool valve. Due to the complex microscopic geometrical features and inherent non-uniform errors of the assembly interface topography, the assembly constraints of precision spool valves will deviate from the theoretical state, leading to non-uniform assembly clearances between the constrained assembly interfaces. The key to study the mating performance of precision spool valves is to grasp the distribution pattern of the above-mentioned non-uniform assembly clearances. Nevertheless, the CAD model of the spool valve constructed in the current design stage takes only ideal assembly into consideration, without being able to reflect the actual assembly constraint state and parameters. Hence, analyzing the mating behavior precision spool valve based on the geometrical model of the assembly interface topography is of great significance to virtual-real mapping and the construction of the digital twin spool valve.

(3) This paper analyzed the influences of non-uniform assembly clearances on the leakage features of precision spool valve, and established the quantitative correlation between mating parameters and the amount of oil leakage. Through theoretical deduction, researchers have developed corresponding equations to theoretically calculate the oil leakage of precision parts, but most of the calculation models assume that the assembly interface has ideal topography features. However, the microscopic topography features and the non-uniform assembly clearances of the assembly interface can greatly influence key parameters such as the diameter of annular clearances, the cross-sectional area and eccentricity of effective clearances, which may reduce the prediction accuracy of the calculation model established based on ideal topography features of the assembly interface. Thus, it is necessary to further expand the classical theoretical equation by considering the influences of the assembly interface topography and incorporating more practical mating parameters into the equation, so as to improve the model's accuracy of predicting oil leakage.

Based on the research framework shown in Figure 1, this paper modeled the geometrical topography of the assembly interface of precision spool valve, modeled the mating behavior of precision spool valves and analyzed the mating performance of precision spool valves.

\section{Digital Twin Modeling of Precision Spool Valve}

\subsection{Overall Outline}

The goal of digital twin modeling is to represent the geometrical topography of the assembly interface. As shown in Figure 2, it is implemented in the following three aspects:

(1) With the CAD model as the basis, this paper extracted the ideal geometrical model and constraint information of the assembly interface. Then, the normal assembly interface is subdivided by the tessellation method so as to discretize the assembly interface based on triangular mesh. Moreover, topography errors of the assembly interface are expressed by mesh and point cloud, which include size errors, position errors and form errors. That is to say, the topography errors mentioned in this paper are a combination of size errors, position errors and form errors. The strength of discretization lies in its ability to satisfy the requirements of constructing the digital twin spool valve by providing a basis for simulating the assembly interface topography in the design stage and offering an interface for building the surface topography based on the measurement point cloud.

(2) By analyzing the design tolerance of the parts, this paper constructed a tolerance zone constraining the variation of topography error of the assembly interface. As we known, the tolerance determines a tolerance zone, which limits the range of topography errors. Meanwhile, it is unrealistic to obtain the topography errors of each part in the manufacturing stage. Therefore, obtaining the topography errors through the tolerance zone is a typical idea. Based on the tolerance zone, a variation constraint equation of mesh points of the assembly interface was established to transform the tolerance zone into the variation range of discrete points, thereby forming the multi-scale topography error height fields of the assembly interface, including the height fields of size error, roughness, waviness and form error. 
(3) Based on NURBS curve and the surface modeling theory, various errors or height fields are fused with original geometric features, namely the theoretical surface, to form the geometrical model of the assembly interface topography. On this basis, the topography parameters of the assembly interface were calculated, and the spatial distribution characteristics of topography errors of the assembly interface are obtained, which laid a foundation for analyzing the mating behavior of the assembly interface.

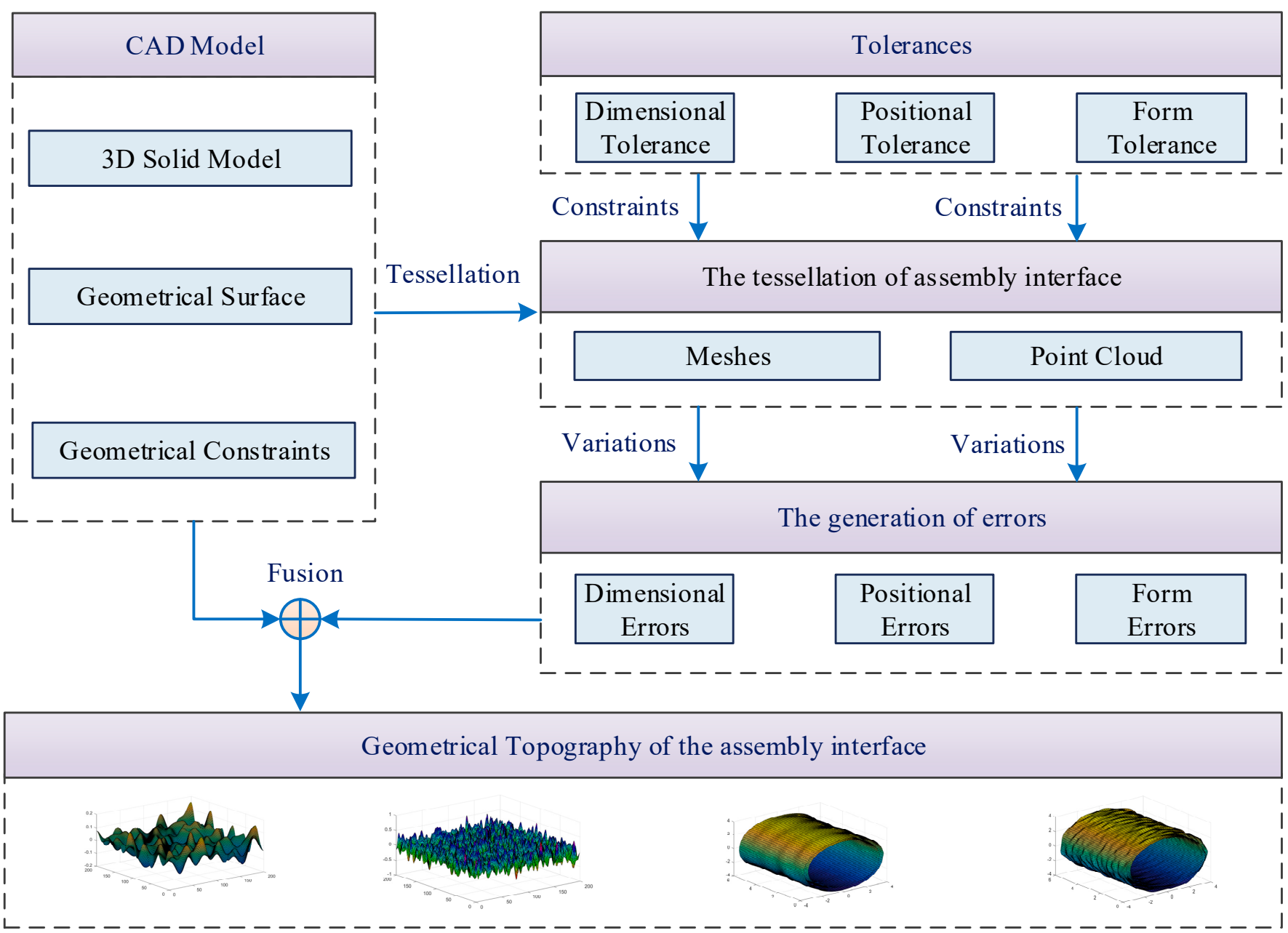

Figure 2. Overall Outline of modeling geometrical topography of the assembly interface.

\subsection{Key Methods}

In view of the research framework proposed in Section 3.1, researchers have proposed a variety of methods to model geometrical topography of the assembly interface, such as parametric surface deformation method [4,5], skin model shapes [6-8] and fractal geometrical method [2,3]. Skin model shapes is one of the most concerned methods in the field of assembly error modeling. Discrete Cosine Transform (DCT), as a typical method to construct skin model shapes, transforms complicated shapes into the linear superposition of a series of orthogonal basis functions. According to the study carried out by the reference [29], orthogonal to each other, the trigonometric functions generated by Fourier transform can serve as the basis functions for constructing the skin model shapes. Hence, the kernel functions are defined as follows:

$$
\left\{\begin{array}{l}
g(m, n, u, v)=\frac{2}{\sqrt{M \times N}} C(u) C(v) \cos \frac{(2 m+1) u \pi}{2 M} \cos \frac{(2 n+1) v \pi}{2 N} \\
C(u)=\left\{\begin{array}{c}
\frac{1}{\sqrt{2}} \text { when } u=0 \\
1 \text { when } u \neq 0
\end{array}, C(v)=\left\{\begin{array}{c}
\frac{1}{\sqrt{2}} \text { when } v=0 \\
1 \text { when } v \neq 0
\end{array}\right.\right.
\end{array}\right.
$$


where $m, u=0,1, \cdots, M-1$ and $n, v=0,1, \cdots, N-1 ; m$ and $n$ denote the number of the sampling points, $M$ and $N$ represent the total quantity of sampling points, $u$ and $v$ represent the frequency value. By substituting each combination of $u$ and $v$ in Equation (1), the basis functions of DCT are formed. According to features of the kernel functions of DCT, the quantity of basis functions is decided by the quantity of sampling points, with dimension of $M \times N$. For instance, if the quantity of sampling points was $200 \times 200$, there will be 40,000 basis functions, with base shapes of different complexity given in Figure 3 . The topography to be characterized is expressed by a linear superposition of the obtained base shapes as follows:

$$
P_{m, n}=\sum_{u=0}^{M-1} \sum_{v=0}^{N-1} \lambda_{u, v} g(m, n, u, v)
$$

where $S_{m, n}$ denotes the height of the sampling point with the number being $(m, n)$ on the surface, and the heights of all sampling points constituted a topography error field; $\lambda_{u, v}$ represents the weight of the basis function numbered as $(u, v)$. In practical application, a large quantity of measurement points usually corresponds to a large quantity of basis functions. However, not all basis functions are needed to characterize the shape of a surface. Instead, the basis functions needed are selected according to the shape complexity of the surface to be characterized and the requirements for details. Specifically, the weight and statistical characteristics of $\lambda_{u, v}$ can be obtained by statistically analyzing multiple batches of data measured on the surface. Based on the original DCT analysis, it has three advantages. Firstly, previous studies have shown that the base shape corresponding to the basis function is closely correlated with the error source in the machining process. Hence, it is practical to reconstruct the geometrical error model with the linear combination of this basis function, which can effectively support the digital twinning process of the precision spool valve. Secondly, the combination of basis functions is flexibly enough to form the geometrical topography of any shape, making basis function strongly applicable to engineering applications. Thirdly, it supports the construction of surface geometrical topography based on actual measurement data, that is, by analyzing the actual measurement data, the statistical characteristics concerning the weight of the base shape (basis function) can be obtained, which makes the geometrical topography closer to the actual process as well as supports virtual-real mapping and interaction.

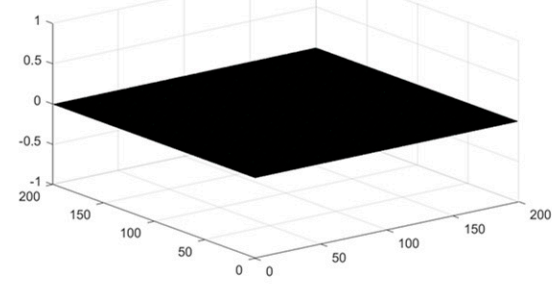

$u=0, v=0$

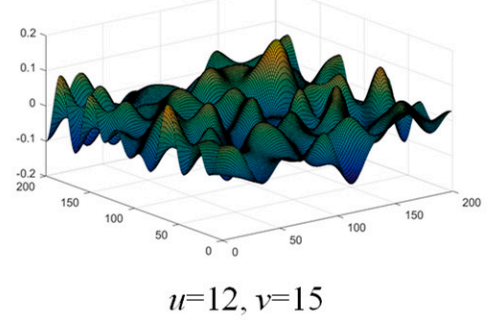

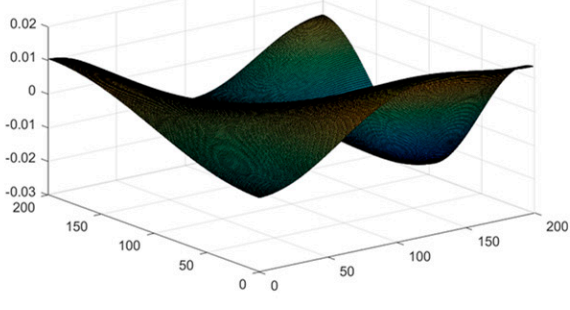

$u=3, v=3$

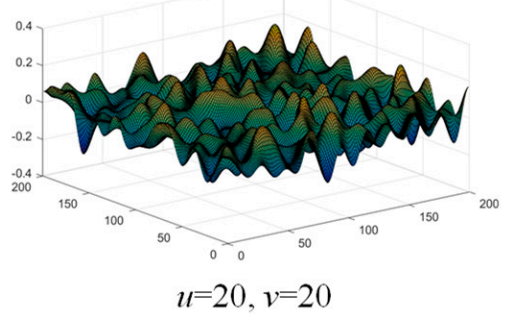

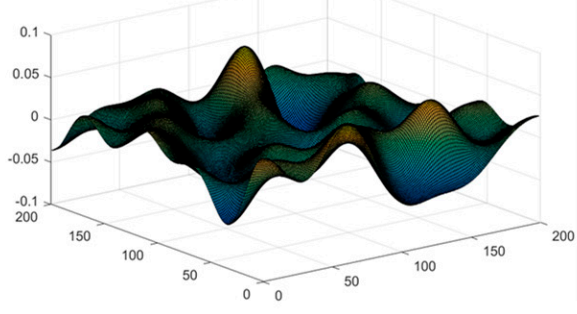

$u=8, v=8$

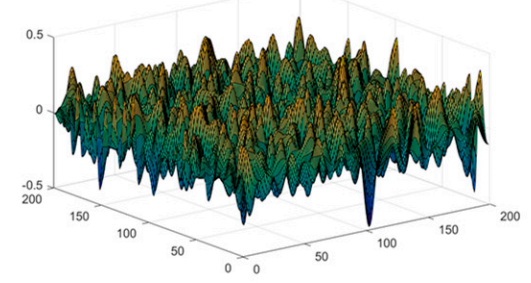

$u=20, v=50$

Figure 3. Base shapes of different complexity. 


\section{Mating Behavior Modeling of Precision Spool Valve Based on Digital Twin \\ 4.1. Overall Outline}

With the geometrical topography of the assembly interface as the basis, this paper conducted modeling and performance analysis of mating behavior of precision spool valve, as shown in Figure 4.

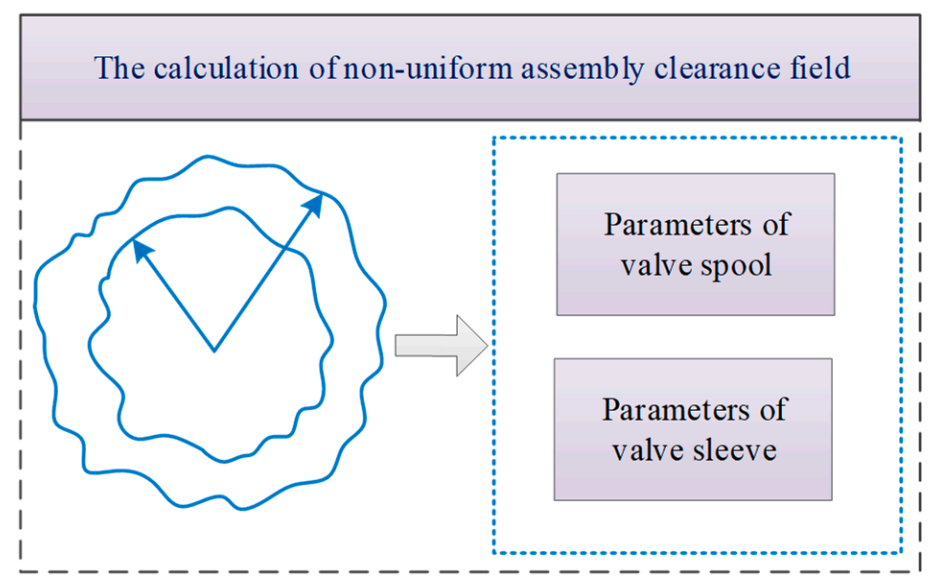

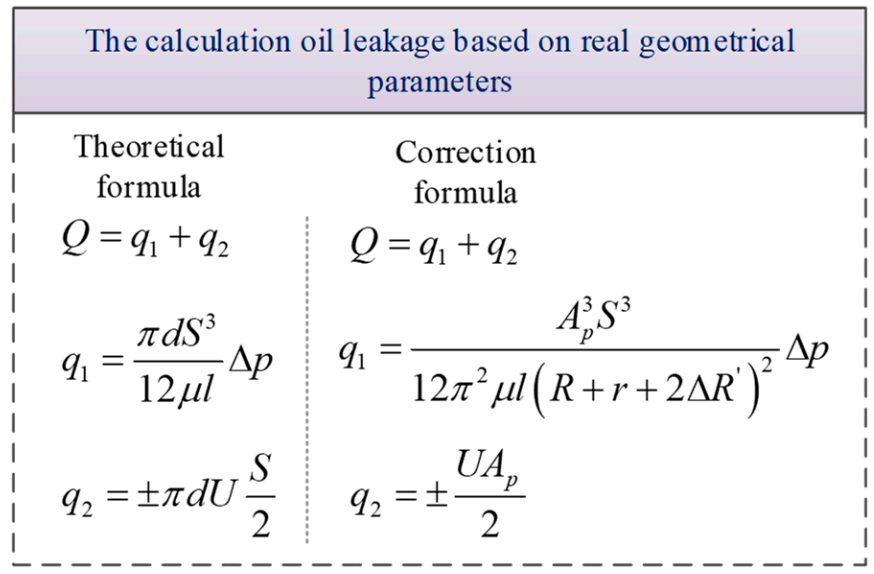

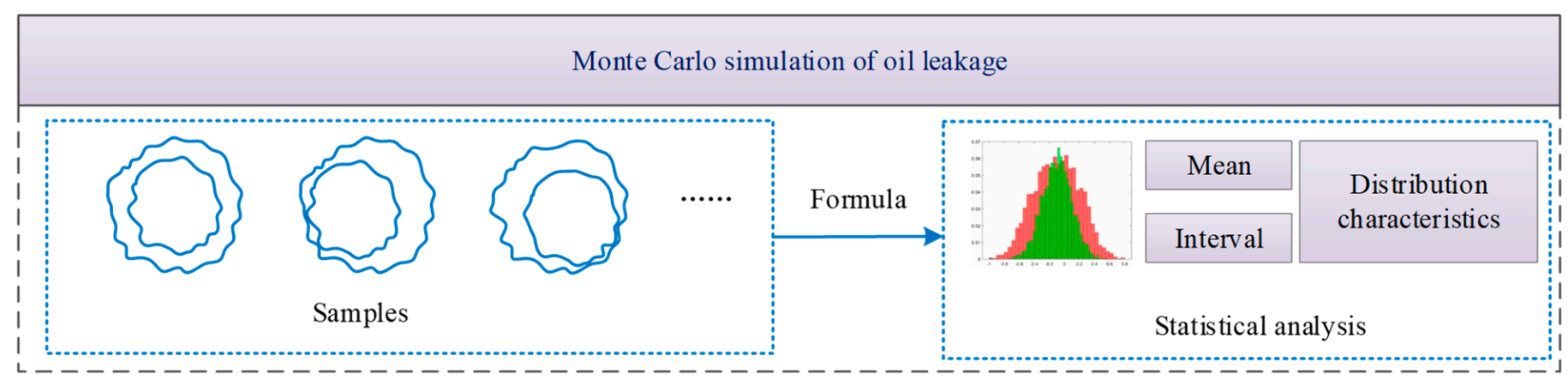

Figure 4. Overall Outline of modeling and performance analysis of mating behavior.

(1) The assembly interfaces with complicated topography characteristics are matched to form the non-uniform assembly clearance field of precision spool valve, based on which the real geometric parameters of valve spool and valve sleeve are analyzed. These parameters include the maximum, minimum, average and distribution parameters of the outer diameter of the valve spool, as well as the maximum, minimum, average and distribution parameters of the inner diameter of valve sleeve. Based on the above parameters, this paper analyzed the parameters indicating the matching between valve spool and valve sleeve, including minimum clearance, maximum clearance, equivalent clearance, eccentricity, coverage, etc., which served as the input parameters to predict the oil leakage rate.

(2) Based on the above-mentioned geometrical parameters, with the classical equation for calculating the oil leakage rate of annular clearance as case, this paper explored a theoretical equation for calculating oil leakage based on real geometrical parameters by replacing the theoretical parameters (e.g., the diameter and length of annular clearance) in the classical equation with real geometrical parameters, thereby expanding the application scope of the classical equation for calculating the oil leakage rate of annular clearance.

(3) The Monte Carlo method was used to simulate different situations of the nonuniform assembly clearance field. In the field of tolerance analysis, Monte Carlo is widely used. This method simulates a large number of actual processed parts through random sampling. After that, by assembling the parts with errors together, the assembly clearances are analyzed [30]. Different clearances will cause the precision spool valve to have different oil leakage. Therefore, in order to comprehensively evaluate the influence of the surface topography error on the oil leakage of the precision spool valve, we introduce the Monte 
Carlo method into the calculation of the oil leakage. Based on the equation for calculating oil leakage, this paper analyzed the amounts of oil leaked under various conditions, studied the statistical parameters (e.g., average, range and distribution) of oil leakage, and grasped the statistical characteristics of oil leakage with a given specified design tolerance.

\subsection{Key Methods}

According to the implementation ideas shown in Figure 4, it is crucial to deduce the oil leakage calculation equation considering the influence of topography characteristics. Based on the classical equation for calculating the oil leakage rate of annular clearance, the oil leakage caused by the differential pressure between the valve spool and valve sleeve can be calculated using the following equation:

$$
q_{1}=\frac{\pi d S^{3}}{12 \mu l} \Delta p
$$

where $q_{1}$ denotes the oil leakage rate of the assembly clearances; $d$ refers to the outer diameter of the valve spool; $S$ refers to the radial clearance; $\mu$ represents dynamic viscosity of the oil; $l$ denotes the length of clearance; $\Delta p$ refers to the pressure difference.

Considering the displacement change of the valve spool during the operation, the oil leakage caused by the movement of the valve spool relative to the valve sleeve can be expressed by the following equation:

$$
q_{2}= \pm \pi d U \frac{S}{2}
$$

where $U$ denotes the speed of relative movement between the valve spool and valve sleeve; when the valve spool moves relative to the valve sleeve along the same direction of oil leakage, the value will be given the plus sign, otherwise the minus sign will be given.

Considering the oil leakage of the above two parts, this paper gained the total amount of oil leakage, which is expressed as follows:

$$
Q=\pi d\left(\frac{S^{3}}{12 \mu l} \Delta p \pm U \frac{S}{2}\right)
$$

In Equation (5), the radial clearance is the difference between the radii of two ideal cylinders, but the value is variable due to the complicated topography characteristics of the surface. Reference [3] expanded the equation by calculating the oil leakage with the cross-sectional area formed by the assembly clearance between valve spool and valve sleeve. On this basis, the equivalent clearance is defined as follows:

$$
S=\frac{A}{\pi d}
$$

where $A$ denotes the area formed by the assembly clearance on a specific cross section. By substituting Equation (6) into Equation (5), the equation considering oil leakage is obtained as follows:

$$
Q=\frac{A \Delta p}{12 \pi^{2} \mu l d^{2}} \pm \frac{U A}{2}
$$

It should be noted that a precision spool valve can have several cross sections, and the equivalent clearance of each cross section can be obtained. This paper selected the minimum equivalent clearance for calculation.

In Equation (7), $l \rightarrow 0, Q \rightarrow \infty$, which greatly differs from the practical situation. Therefore, when $l \leq 0.2 \mathrm{~mm}$, the following empirical equation can be used [31].

$$
Q=\frac{0.87 \pi d\left(W^{2}+S^{2}\right)^{2 / 3} \Delta p^{\frac{2+a W W^{2}}{2+2 a W^{2}}}}{60 \gamma\left(1+2.94 \times 10^{-3} l^{3 / 2}\right)}
$$


where $W$ denotes the positive opening of the valve port; $a$ is a constant, $a=7.5 \times 10^{-4} ; \gamma$ represents the mass of the oil per unit volume.

\section{Example Verification and Results}

\subsection{A Case of Precision Spool Valve}

As shown in Figure 5, the precision spool valve consists of a valve spool and a valve sleeve. The main technological requirements are as follows: the valve spool with a diameter of $\varnothing 6.6 \mathrm{~mm}$ and the valve sleeve with an aperture of $\varnothing 6.6_{0}^{+0.015} \mathrm{~mm}$ are processed and assembled to ensure that the assembly clearance size ranged from $0.002 \mathrm{~mm}$ to $0.004 \mathrm{~mm}$; the measured sizes A and B of the valve sleeve are matched with the match grinding sizes $\mathrm{a}$ and $\mathrm{b}$ of valve spool, so that the overlapped part could vary from $0.010 \mathrm{~mm}$ to $0.015 \mathrm{~mm}$; the cylindricity tolerance of the valve spool's inner surface was $0.003 \mathrm{~mm}$, and that of the valve sleeve's outer surface was $0.001 \mathrm{~mm}$; the red arrow indicates the direction along which the oil is supplied, with $P_{1}, P_{2}, P_{3}$ and $P_{4}$ representing the corresponding oil supply pressures; in the initial state, $P_{1}=P_{3}=21 \mathrm{MPa}, P_{2}=P_{4}=10.5 \mathrm{MPa}$; as the valve spool moved right, $P_{2}$ increases linearly from $10.5 \mathrm{MPa}$ to $21 \mathrm{MPa}$, while $P_{4}$ drops linearly from $10.5 \mathrm{MPa}$ to 0 ; when the valve spool moves left, $P_{2}$ and $P_{4}$ changes oppositely; $P_{1}$ and $P_{3}$ remains constant, and one movement stroke of the valve spool is $0.68 \mathrm{~mm}$. All oil outlets are assumed to be closed in the analysis of oil leakage.

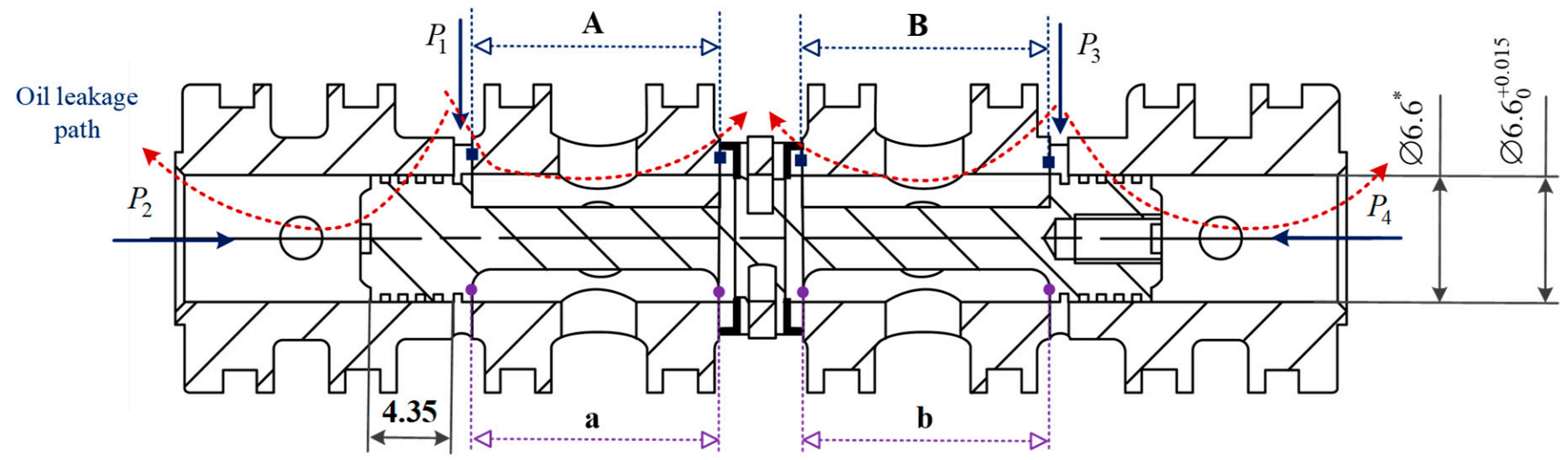

Figure 5. Assembly diagram of precision spool valve.

\subsection{Modeling of Geometrical Topography of the Assembly Interface}

The method proposed in Section 3 is used to construct the geometrical morphology of the assembly interface of precision spool valve, as shown in Figure 6 after being enlarged by 100 times. The geometrical topography is modeled as follows: first of all, the assembly interface is extracted from the CAD model of precision spool valves and then discretized; secondly, in view of the dimensional tolerance and cylindricity tolerance of assembly interface, the DCT method is used to establish the error field of each discrete point on the assembly interface; in the end, the error field is fused with the original assembly interface to construct the geometrical topography. This paper applied the Monte Carlo method to generate a large number of qualified spool valves in different shapes and form digital twin spool valve models, thereby providing more comprehensive and accurate input for analyzing the oil leakage features of spool valves in the design stage [30]. 


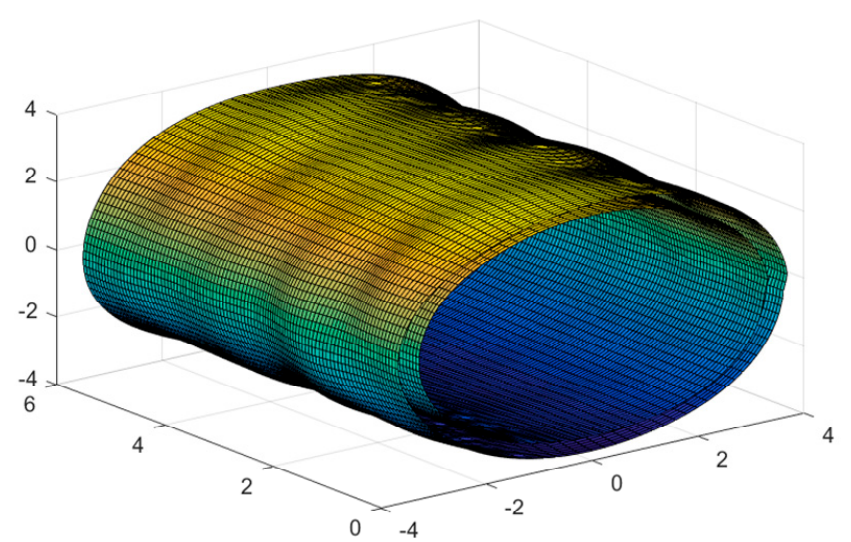

Case 1

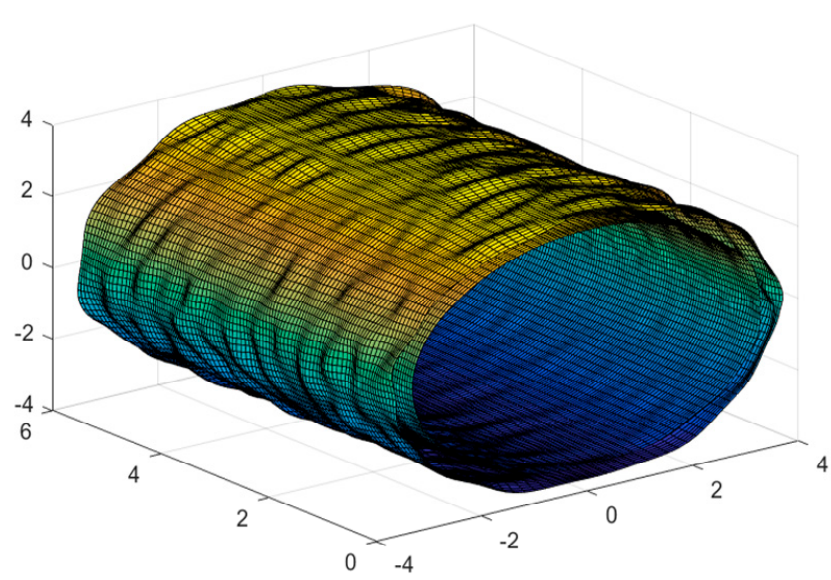

Case 3

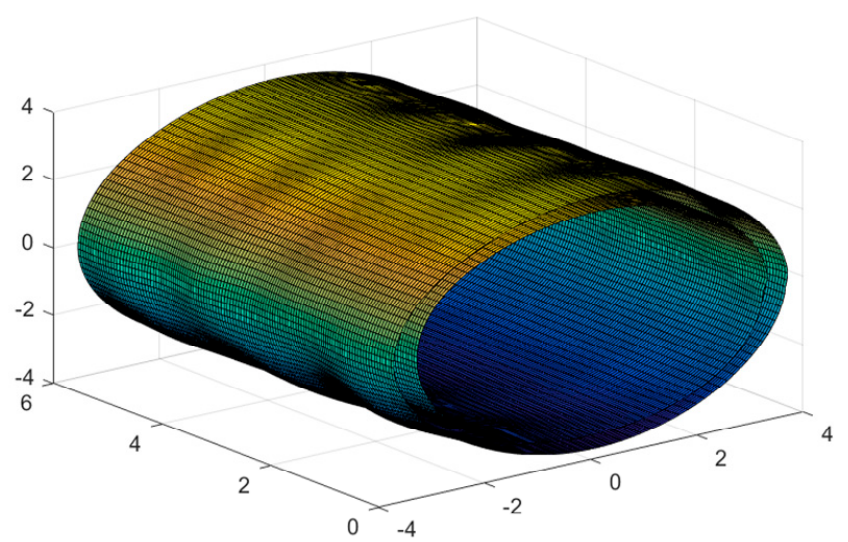

Case2

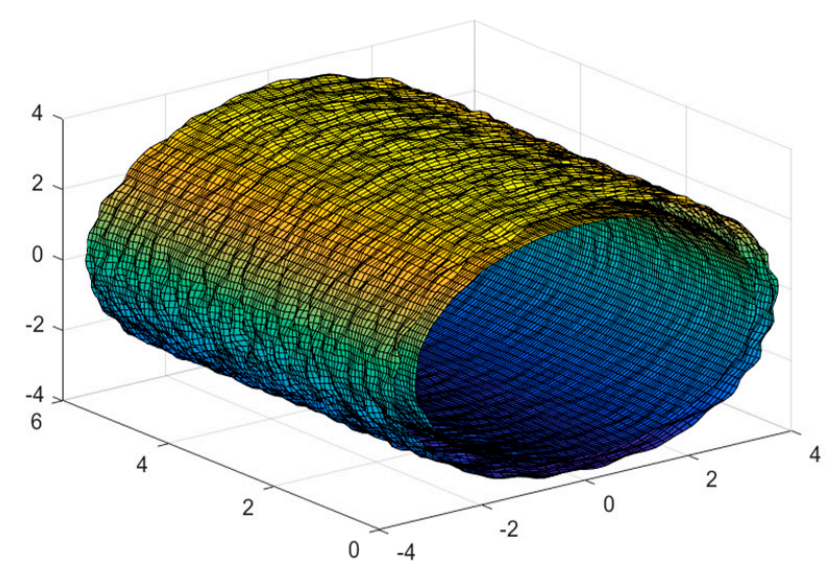

Case 4

Figure 6. Geometrical topography of the assembly interface of precision spool valve.

\subsection{Analysis of Mating Performance}

The mating performance of this spool valve is analyzed based on the parameters shown in Figure 5. Without considering the oil leakage caused by the movement speed of the valve spool, two samples with a clearance of $0.002 \mathrm{~mm}$ are randomly generated for analysis. Figure 7 shows two curves of oil leakage varying with the movement distance of the valve spools. Likewise, two samples are randomly generated with a clearance of $0.003 \mathrm{~mm}$ after processing and assembly, and through analysis, two curves of oil leakage varying with the movement distance of the valve spools are obtained as shown in Figure 8 . It is noted that Case1 and Case 2 in Figure 7 are randomly generated according to the tolerances. It is the same for Case 3 and Case 4 . In actual production, parts are processed according to tolerances, so the above-mentioned four cases may correspond to four instances in engineering.

As shown in Figures 7 and 8, from the zero point, the oil leakage rate keeps increasingly constantly and reached its peak when the movement distance of valve spool ranged from $0.010 \mathrm{~mm}$ to $0.015 \mathrm{~mm}$. Due to the negative opening of the valve port and enclosed ends of the spool valve at zero point, the oil leakage is in a laminar state according to the Bernoulli equation $[3,13]$. When the spool valve moved to a distance of $0.010-0.015 \mathrm{~mm}$, there is positive opening at one end of the spool valve and negative opening at the other end, so the oil leakage is in a turbulent state, which applied to Equation (8) [31]. The distance of $0.010-0.015 \mathrm{~mm}$ is determined by the overlap between the valve spool and a valve sleeve, which is determined by the designer. After the peak of oil leakage rate, the valve spool continued to move, but once the movement distance reached $0.2 \mathrm{~mm}$, the oil leakage is in a stable laminar state, which is applicable to Equation (7). 
Leakage Curves with Gap 0.002mm

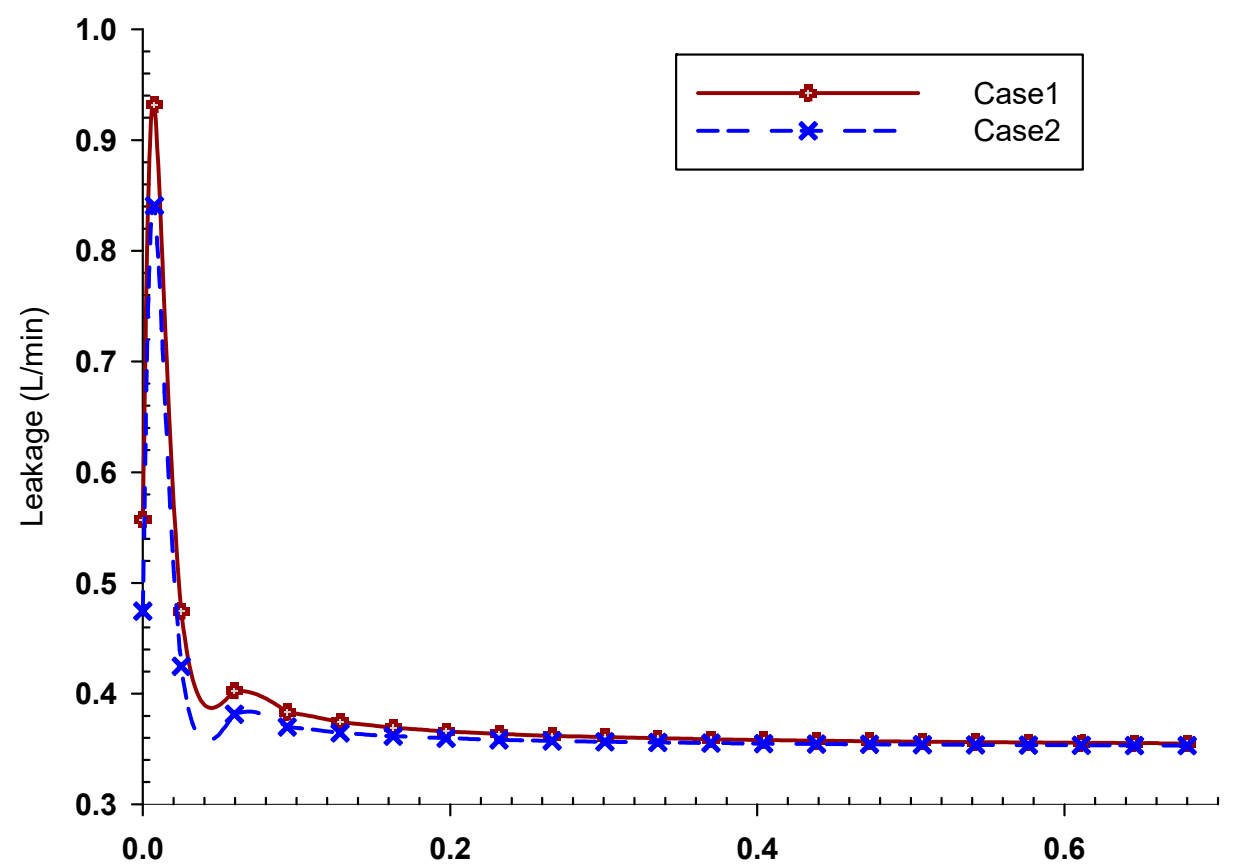

Figure 7. Dynamic curve of oil leakage rate with a clearance of $0.002 \mathrm{~mm}$.

Leakage Curves with Gap 0.003mm

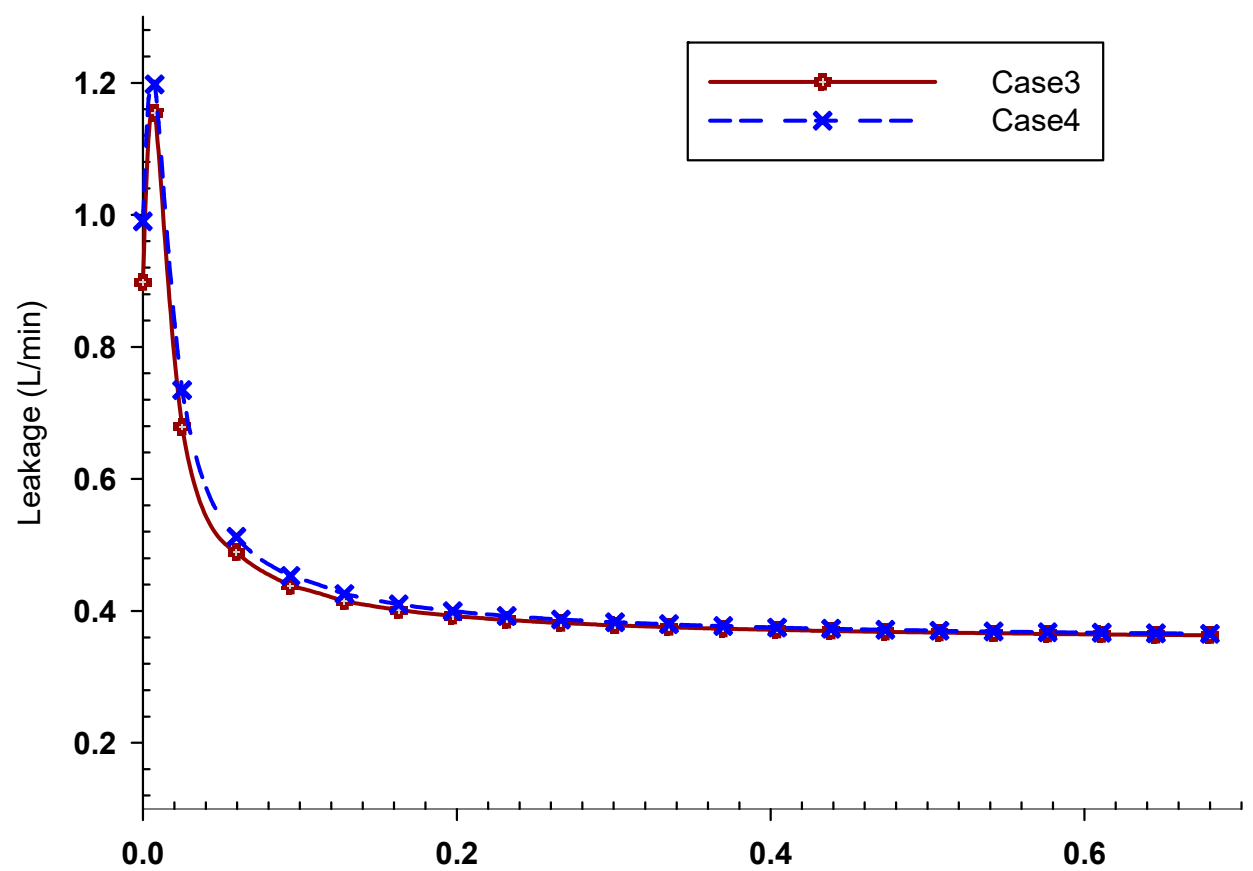

Figure 8. Dynamic curve of oil leakage rate with a clearance of $0.003 \mathrm{~mm}$.

Engineers engaged in the design and manufacturing of spool valves are greatly concerned about zero and peak oil leakage rates [3,31]. In this paper, the Monte Carlo method was used to simulate and analyze the oil leakage rate with a clearance of $0.002 \mathrm{~mm}$ for 2000 times to obtain the statistical distribution charts of the zero oil leakage rate and peak oil leakage rate, as shown in Figures 9 and 10, respectively. According to the 6-Sigma principle, the zero oil leakage rate is within the range of $[0.3413,0.6635]$, and the peak oil leakage rate ranged from 0.6852 to 1.0506 . 


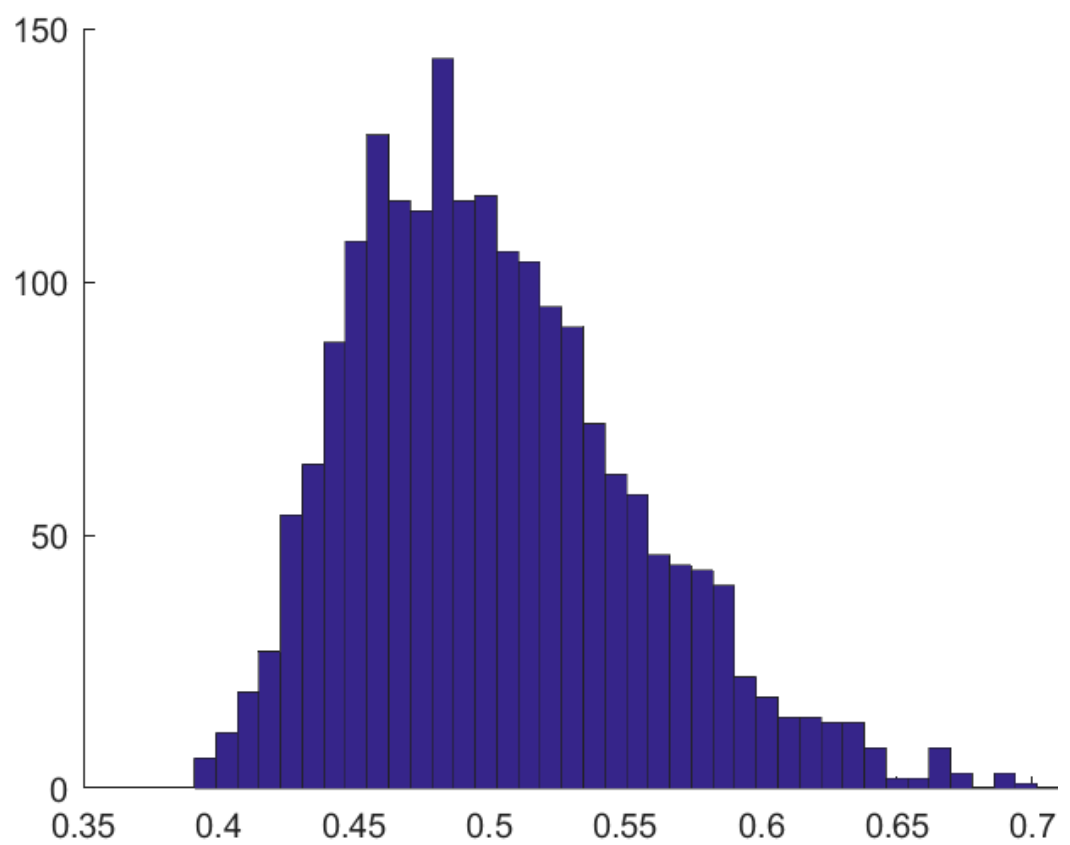

Figure 9. Statistical distribution chart of zero oil leakage rate.

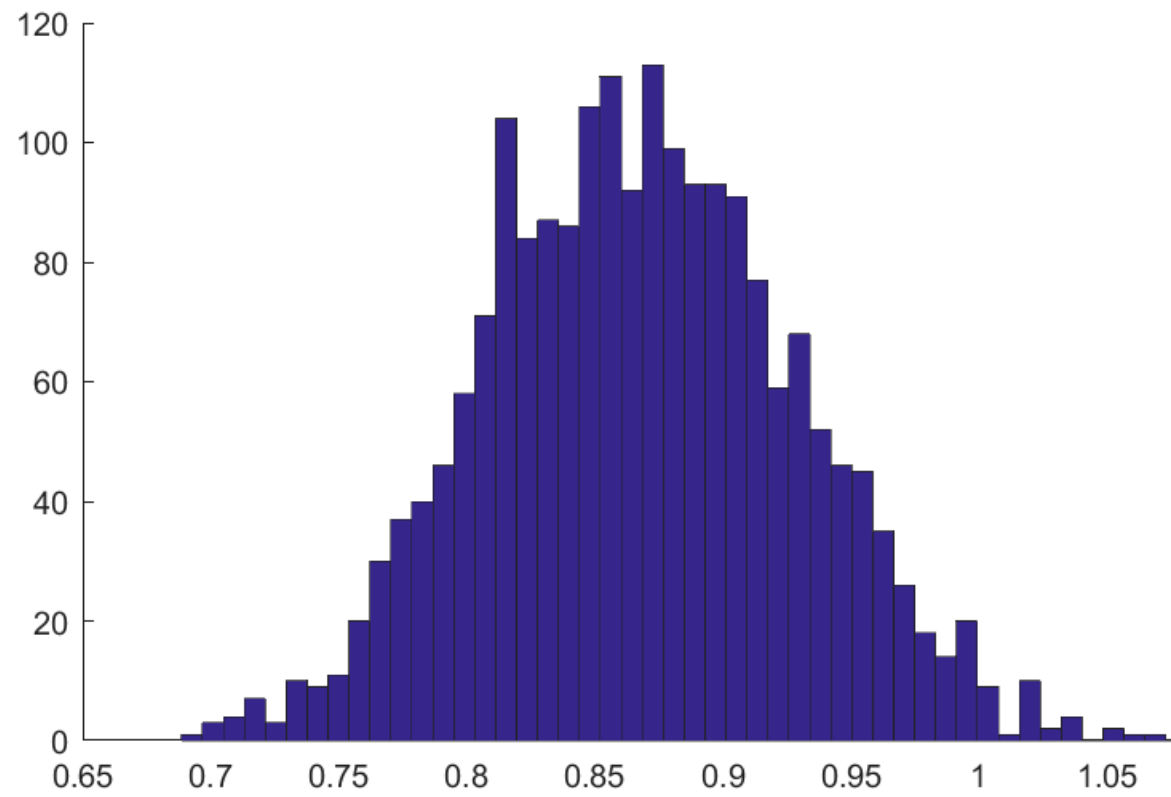

Figure 10. Statistical distribution chart of peak oil leakage rate.

\section{Conclusions}

A precision spool valve is the core component of the electro-hydraulic servo control system. The performance and reliability of precision spool valves exert great influences on the performance and safety of the flight control systems of aviation and aerospace products. This paper proposed a new method to analyze the assembly performance of the precision spool valve based on the digital twin technology. Based on the fivedimensional digital twin framework, this paper established an overall framework to analyze the assembly performance of precision slide valves based on a digital twin, pointing out the key technologies. The procedures and methods of modeling geometrical topography of the assembly interface of the precision spool valve were proposed to characterize the complicated topography errors of assembly interface of the precision spool valve. Based on the concept of Skin Model Shapes, the geometrical topography of assembly interface was constructed. This paper analyzed the mating behavior of precision spool 
valves with the influence of topography errors taken into consideration, established the quantitative relationship between non-uniform assembly clearance and oil leakage rate and quantitatively evaluated the assembly performance of precision spool valves from the perspective of oil leakage. Using the quantitative model, it was easily to evaluate the mating performance of precision spool valves. Designers may use the quantitative model to evaluate the performance of precision spool valves in design phase or in manufacturing phase. The proposed methods in this paper were validated by a precision spool valve, and the results show the feasibility. Guided by the digital twin framework, this paper combined digital twin data with the modeling of geometrical topography and mating behavior of precision spool valves, as well as the analysis of mating performance of the precision spool valve to simulate, analyze and predict the mating behavior and performance of physical entities of precision spool valves, which contributes to the optimization decision making concerning the design, processing, assembly and adjustment of precision spool valves. Based on the research results gained in this paper, it is necessary to further explore how to optimize and control the mating performance of precision spool valves by adjusting the geometrical topography parameters and assembly parameters of the assembly interface.

Author Contributions: Conceptualization, W.T. and G.X.; Methodology, W.T.; Validation, S.Z. and S.J.; Writing-original draft, W.T. and R.W.; Writing—review and editing, S.J. All authors have read and agreed to the published version of the manuscript.

Funding: This research was funded by "the Natural Science Foundation of Shaanxi Province, grant number 2021JQ-680", "the Open Fund of the State Key Laboratory for Manufacturing Systems Engineering of China, grant number sklms2020003", "the National Key Research and Development Program, grant number 2019YFB1707205-1", "the Scientific Research Foundation for Doctor of Xi'an Polytechnic University, grant number BS201909" and "the Shaanxi Key Research and Development Program, grant number 2020GY-172".

Data Availability Statement: Not applicable.

Conflicts of Interest: The authors declare no conflict of interest.

\section{References}

1. Liu, Q.Y.; Lu, C.; Liu, T.; Xu, Z.B. Adaptive Cutting Control for Roadheaders Based on Performance Optimization. Machines 2021, 9, 46. [CrossRef]

2. Qiu, C.; Liu, Z.Y.; Bu, W.H.; Tan, J.R. Hybrid dimension based modeling of part surface topography and identification of its characteristic parameters. Appl. Surf. Sci. 2012, 258, 7082-7093. [CrossRef]

3. Qiu, C.; Liu, Z.Y.; Peng, X.; Tan, J.R. A Non-Ideal Geometry Based Prediction Approach of Fitting Performance and Leakage Characteristic of Precision Couplings. IEEE Access 2018, 6, 58204-58212.

4. Luo, C.; Franciosa, P.; Ceglarek, D.; Ni, Z.; Jia, F. A novel geometric tolerance modeling inspired by parametric space envelope. IEEE Trans. Autom. Sci. Eng. 2018, 15, 1386-1398. [CrossRef]

5. Zhang, Z.Q.; Zhang, Z.J.; Jin, X.; Zhang, Q.S. A novel modelling method of geometric errors for precision assembly. Int. J. Adv. Manuf. Technol. 2018, 94, 1139-1160. [CrossRef]

6. Anwer, N.; Ballu, A.; Mathieu, L. The skin model, a comprehensive geometric model for engineering design. CIRP Ann. 2013, 62, 143-146. [CrossRef]

7. Schleich, B.; Wartzack, S. Evaluation of geometric tolerances and generation of variational part representatives for tolerance analysis. Int. J. Adv. Manuf. Technol. 2015, 79, 959-983. [CrossRef]

8. Yan, X.; Ballu, A. Generation of consistent skin model shape based on FEA method. Int. J. Adv. Manuf. Technol. 2017, 92, 789-802. [CrossRef]

9. Sun, Q.C.; Zhao, B.B.; Liu, X.; Mu, X.K.; Zhang, Y.L. Assembling deviation estimation based on the real mating status of assembly. Comput. Aided Des. 2019, 115, 244-255. [CrossRef]

10. Sun, X.M.; Bao, J.S.; Li, J.; Zhang, Y.M.; Liu, S.M.; Zhou, B. A digital twin-driven approach for the assembly-commissioning of high precision products. Robot. Comput. Integr. Manuf. 2020, 61, 101839. [CrossRef]

11. Schleich, B.; Wartzack, S. Novel approaches for the assembly simulation of rigid Skin Model Shapes in tolerance analysis. Comput. Aided Des. 2018, 101, 1-11. [CrossRef]

12. Grandjean, J.; Ledoux, Y.; Samper, S. On the role of form defects in assemblies subject to local deformations and mechanical loads. Int. J. Adv. Manuf. Technol. 2013, 65, 1769-1778. [CrossRef]

13. Mondal, M.K.; Manna, N.K.; Saha, R. Study of leakage flow through a spool valve under blocked-actuator port conditionSimulation and experiment. Proc. Inst. Mech. Eng. Part C J. Mech. Eng. Sci. 2014, 228, 1405-1417. [CrossRef] 
14. Afatsun, A.C.; Balkan, R.T. A Mathematical Model for Simulation of Flow Rate and Chamber Pressures in Spool Valves. J. Dyn. Syst. Meas. Control. 2019, 141, 021004. [CrossRef]

15. Wang, K.; Liu, D.X.; Liu, Z.Y.; Tan, J.R. An assembly precision analysis method based on a general part digital twin model. Robot. Comput. Integr. Manuf. 2021, 68, 102089. [CrossRef]

16. Liu, M.N.; Fang, S.L.; Dong, H.Y.; Xu, C.Z. Review of digital twin about concepts, technologies, and industrial applications. J. Manuf. Syst. 2021, 58, 346-361. [CrossRef]

17. Semeraro, C.; Lezoche, M.; Panetto, H. Digital twin paradigm: A systematic literature review. Comput. Ind. 2021, 130, 103469. [CrossRef]

18. Tao, F.; Anwer, N.; Liu, A.; Wang, L.; Nee, A.Y.; Li, L.; Zhang, M. Digital twin towards smart manufacturing and industry 4.0. J. Manuf. Syst. 2021, 58,1-2. [CrossRef]

19. Cai, H.; Zhu, J.; Zhang, W. Quality Deviation Control for Aircraft Using Digital Twin. J. Comput. Inf. Sci. Eng. 2021, 21, 031008. [CrossRef]

20. Zhang, C.; Sun, Q.; Sun, W.; Mu, X.; Wang, Y. A construction method of digital twin model for contact characteristics of assembly interface. Int. J. Adv. Manuf. Technol. 2021, 113, 2685-2699. [CrossRef]

21. Tao, F.; Sui, F.Y.; Liu, A.; Qi, Q.L.; Zhang, M.; Song, B.Y.; Guo, Z.R.; Lu, S.C.Y.; Nee, A.Y.C. Digital twin-driven product design framework. Int. J. Prod. Res. 2019, 57, 3935-3953. [CrossRef]

22. Liu, S.M.; Lu, Y.Q.; Li, J.; Song, D.Q.; Sun, X.M.; Bao, J.S. Multi-scale evolution mechanism and knowledge construction of a digital twin mimic model. Robot. Comput. Integr. Manuf. 2021, 71, 102123. [CrossRef]

23. Villalonga, A.; Negri, E.; Biscardo, G.; Castano, F.; Haber, R.E.; Fumagalli, L.; Macchi, M. A decision-making framework for dynamic scheduling of cyber-physical production systems based on digital twins. Annu. Rev. Control. 2021, 51, 357-373. [CrossRef]

24. Fang, Y.L.; Peng, C.; Lou, P.; Zhou, Z.D.; Hu, J.M.; Yan, J.W. Digital-Twin-Based Job Shop Scheduling Toward Smart Manufacturing. IEEE Trans. Ind. Inform. 2019, 15, 6425-6435. [CrossRef]

25. Castaño, F.; Haber, R.E.; Mohammed, W.M.; Nejman, M.; Villalonga, A.; Martinez Lastra, J.L. Quality monitoring of complex manufacturing systems on the basis of model driven approach. Smart Struct. Syst. 2020, 26, 495-506.

26. Moretti, M.; Rossi, A.; Senin, N. In-process monitoring of part geometry in fused filament fabrication using computer vision and digital twins. Addit. Manuf. 2021, 37, 101609.

27. Söderberg, R.; Warmefjord, K.; Carlson, J.S.; Lindkvist, L. Toward a Digital Twin for real-time geometry assurance in individualized production. CIRP Ann. 2017, 66, 137-140. [CrossRef]

28. Schleich, B.; Anwer, N.; Mathieu, L.; Wartack, S. Shaping the digital twin for design and production engineering. CIRP Ann. 2017, 66, 141-144. [CrossRef]

29. Liu, J.H.; Zhang, Z.Q.; Ding, X.Y.; Shao, N. Integrating form errors and local surface deformations into tolerance analysis based on skin model shapes and a boundary element method. Comput. Aided Des. 2018, 104, 45-59. [CrossRef]

30. Tang, W.B.; Xu, G.S.; Zhangm, S.J. Dimensional variation analysis for rigid part assembly with an improvement of Monte Carlo simulation. IEEE Access 2020, 8, 5862-5872. [CrossRef]

31. Liu, J.; Sun, S.L.; Liu, Q.D.; Huang, S.Y.; Li, Z.H.; Xin, J.H. Effect of Alignability of Three-position Reversal Spool Valve. Trans. Chin. Soc. Agric. Mach. 2001, 32, 72-74. (In Chinese) 